Psychotherapeut 2018 63:235-242 https://doi.org/10.1007/s00278-018-0271-7 Online publiziert: 30. Januar 2018 (c) Der/die Autor(en) 2018. Dieser Artikel ist eine Open-Access-Publikation.

\section{Redaktion}

Bernhard Strauß, Jena

CrossMark

Hella Parpart ${ }^{1,2} \cdot$ Magdalena Krankenhagen $^{2} \cdot$ Laura Albantakis $^{1,2} \cdot$ Lara Henco $^{1,2}$. Elisabeth Friess ${ }^{3} \cdot$ Leonhard Schilbach $^{1,2,4}$

' Unabhängige Max-Planck-Forschungsgruppe für Soziale Neurowissenschaft, Max-Planck-Institut für Psychiatrie, München, Deutschland

${ }^{2}$ Ambulanz und Tagklinik für Störungen der Sozialen Interaktion, Max-Planck-Institut für Psychiatrie, München, Deutschland

${ }^{3}$ Tagklinik für Psychiatrie und Psychotherapie, Max-Planck-Institut für Psychiatrie, München, Deutschland ${ }^{4}$ Klinik und Poliklinik für Psychiatrie und Psychotherapie, Ludwig-Maximilians-Universität München, München, Deutschland

\title{
Schematherapie-informiertes, soziales Interaktionstraining
}

\section{Interventionsansatz für Erwachsene mit hochfunktionalem Autismus}

\begin{abstract}
Neue Erkenntnisse geben Hinweise darauf, dass Personen mit hochfunktionalem Autismus (HFA) zwar bestimmte soziale Fähigkeiten erlernen und auch bewusst abrufen können, jedoch kommt es bei ihnen nicht zu Veränderungen der automatischen Anpassungsleistungen. Deshalb sollte der Fokus eines Interventionsansatzes auf der Steigerung interaktionsrelevanter sozialer Kompetenzen liegen. Diese kann gelingen, indem mithilfe des Schematherapie-informierten, sozialen Interaktionstrainings (STISI) für Erwachsene die Komplexität sozialer Situationen für HFA-Betroffene reduziert wird.
\end{abstract}

\section{Hintergrund}

Das wissenschaftliche und gesellschaftliche Interesse an Autismus-SpektrumStörungen (ASS) hat in den letzten Jahren stark zugenommen. Neuen epidemiologischen Studien zufolge beträgt die Prävalenzrate für ASS zwischen $1 \%$ und 1,5\% (Developmental DMNSY, 2010 Principal Investigators 2014; Kim et al. 2011; Lyall et al. 2017). Der Anstieg der Prävalenzrate in den letzten 15 Jahren ist insbesondere auf die vermehrte Identifikation erwachsener, autistischer Personen mit hohem Funktionsniveau zurückzuführen (Lyall et al. 2017). Etwa die Hälfte aller Menschen mit ASS wird als hochfunktional eingestuft (Developmental DMNSY, 2010 Principal Investigators 2014). Ein Teil der Personen mit HFA erreicht das Erwachsenenalter, ohne dass eine Diagnose in Kindheit und Jugend gestellt wurde. Aufgrund einer meist hohen Intelligenz können sozial-kommunikative und -interaktionelle Defizite durch kognitive Lernprozesse kompensiert werden (Lehnhardt et al. 2011). Schwierigkeiten zeigen sich meist erst in Schwellensituationen oder Transitionsphasen (z. B. beim Übergang in ein neues soziales Leben nach der Schulzeit), die erhöhte Anforderungen an die soziale Kompetenz stellen und in denen die gelernten Kompensationsstrategien nicht helfen. Frustration, Stress und komorbide psychische Erkrankungen (Lugnegård et al. 2011) sind häufig die Folge. Etwa $70 \%$ der Erwachsenen mit ASS haben komorbide Erkrankungen (Lehnhardt et al. 2013), die auch der Hauptgrund dafür sind, dass erwachsene Personen mit HFA Hilfe suchen (Gaus 2007). Bislang ist noch ungeklärt, welche Form der Unterstützung und Hilfe für diese Personen am förderlichsten ist. Die aktuell vorhandenen psychotherapeutischen Maßnahmen für Erwachsene mit HFA (Ebert et al. 2013; Gawronski et al. 2012) beruhen auf Konzepten der
Verhaltenstherapie und konzentrieren sich neben psychoedukativen Elementen auf den Umgang mit Stress sowie den Ausbau kommunikativer und sozialer Fähigkeiten (Krämer et al. 2016). Jedoch steht eine Validierung dieser Gruppentherapiekonzepte noch aus, sodass keine spezifischen Wirkfaktoren benannt werden können.

Neuere Arbeiten im Bereich der sozialen Neurowissenschaften und Experimentalpsychologie geben Hinweise darauf, dass bei Personen mit HFA eine Dissoziation zwischen impliziten und expliziten sozialen Fähigkeiten vorliegt. Zwar können bestimmte soziale Fähigkeiten erlernt und bewusst abgerufen werden, jedoch kommt es nicht zu Veränderungen von automatischen Anpassungsleistungen im sozialen Kontext (Schilbach 2016). Deshalb sollte der Fokus eines Interventionsansatzes für Personen mit HFA weniger auf der Vermittlung von sozialen Wahrnehmungsleistungen, sondern eher auf der Steigerung interaktionsrelevanter sozialer Kompetenzen liegen. In diesem Beitrag wird das Modusmodell der ST als Basis eines Interaktionstrainings für Erwachsene mit HFA vorgestellt. Die Autoren schlagen vor, dass über die Erkennung von Bewältigungsmodi bei nichtautistischen Interaktionspartnern eine Komplexitätsreduktion sozialer Situationen sowie ein 
besseres Verständnis für interaktionelle Schwierigkeiten erreicht werden können.

\section{Schematherapiekonzept}

\section{Zentrale Bausteine}

Die ST stellt einen integrativen Psychotherapieansatz nach Young et al. (2008) dar, der Techniken der kognitiven Verhaltenstherapie, der psychodynamischen, der Gestalt- und der interpersonellen Psychotherapie sowie der Bindungstheorie vereint. Die ST wird häufig der „dritten Welle“ der Verhaltenstherapie zugeordnet (Hayes 2004), da sie einen Schritt weiter als die kognitive Therapie geht und nicht nur Kognitionen in den Fokus der Therapie stellt (Beck et al. 2004), sondern emotionale Prozesse als zentrales Merkmal und Leitfaden der therapeutischen Arbeit beschreibt. Mehrere randomisierte, kontrollierte Studien weisen auf eine gute Wirksamkeit der ST hin (Bamelis et al. 2015).

Young et al. (2008) definieren eine sichere Bindung, Autonomie, Selbstkontrolle, Freiheit im Ausdruck von Bedürfnissen und Emotionen sowie Spontanität und Spiel als emotionale Grundbedürfnisse. Werden diese durch negative Erfahrungen in Kindheit und Jugend frustriert, entstehen so nimmt die ST an - neuronal verankerte Erlebnisschablonen bzw. maladaptive „Schemata“ („early maladaptive schemas“, EMS) wie „Unzulänglichkeit/ Scham“ oder „unerbittliche Ansprüche“. Diese neuronale Erregungsbereitschaft wird als neuronale Basis eines Schemas angenommen (Roediger 2016). Erst im aktivierten Zustand wird das schemaassoziierte Erleben von Emotionen, Gedanken, Körperempfindungen und Handlungstendenzen sichtbar, was als Schemamodus bezeichnet wird. Diese Schemamodi können im Vergleich zu den eigentlichen Schemata besser beobachtet und direkt angesprochen werden. Stabile und anhaltende EMS werden als Kern der chronischen Achse-I- und Achse-II-Störungen betrachtet. Ziel der ST ist es, den Einfluss der EMS zu reduzieren und die dysfunktionalen CopingStile durch flexiblere und adaptivere Ver- haltensweisen in sozialen Interaktionen zu ersetzen.

\section{Grenzen oder Chancen}

Autismus-Spektrum-Störungen werden in der 10. Auflage der Internationalen statistischen Klassifikation der Krankheiten und verwandter Gesundheitsprobleme (ICD-10; Weltgesundheitsorganisation 2013) in die Kategorie tiefgreifende Entwicklungsstörungen eingeordnet, die sich im Kindesalter manifestieren und remissionslos verlaufen. Im Wesentlichen wird zwischen dem frühkindlichen $\mathrm{Au}$ tismus (F84.0), dem Asperger-Syndrom (AS; F84.5) und dem atypischen Autismus (F84.1) unterschieden. Der HFA beschreibt Individuen mit frühkindlichem Autismus, d.h. mit einer verzögerten Sprachentwicklung, die mindestens eine durchschnittliche Intelligenz aufweisen. Ist die kognitive Leistungsfähigkeit unbeeinträchtigt und liegt eine unauffällige Sprachentwicklung vor, wird die Diagnose AS vergeben. Da diese Sprachentwicklungsstörung aber im Erwachsenenalter zu keinem relevanten Unterschied in der sozialen Anpassungsleistung führt, kann der Einfachheit halber vom HFA als übergreifende, das AS einschließende Kategorie gesprochen werden.

Alle Formen des Autismus mit ihrer Vielzahl an Symptomen und unterschiedlichen Ausprägungsgraden umfassen 3 Kernkriterien: (a) Beeinträchtigungen in sozialer Interaktion, (b) Störungen der Kommunikation sowie (c) stereotype Interessen und Verhaltensweisen. Da eine valide Unterscheidung der 3 Unterformen empirisch nur schwer nachweisbar ist (Frazier et al. 2012; KampBecker et al. 2010), werden diese in der 5. Ausgabe des Diagnostic and Statistical Manual of Mental Disorders (DSM-5; American Psychiatric Association 2013) zu den "Autismus-Spektrum-Störungen“" zusammengefasst, sodass die Diagnose Autismus dimensional auf unterschiedlichen funktionalen Niveaus vergeben wird. Sowohl Personen mit HFA als auch mit AS weisen im Erwachsenenalter eine durchschnittliche bis überdurchschnittliche Intelligenz sowie eine funktionale Sprache auf, weshalb eine eindeutige
Abgrenzung beider Störungsbilder bei erwachsenen Betroffenen schwierig ist. Vielmehr werden unterschiedliche Ausprägungsgrade auf einem Kontinuum derselben zugrunde liegenden Beeinträchtigung vermutet (Remschmidt und Kamp-Becker 2006). Der hier vorgestellte Behandlungsansatz richtet sich an hochfunktionale Erwachsene mit einer ASS und adressiert somit sowohl Personen mit AS als auch HFA.

Early maladaptive schemas werden als Risikofaktoren für unterschiedliche psychiatrische Erkrankungen angesehen (Barazandeh et al. 2016; Bortolon et al. 2013; Kim et al. 2014; Nilsson et al. 2015). Wie lässt sich nun das Schemakonzept, das die Ausbildung maladaptiver Schemata in der Kindheit annimmt und diese als Ursache klinischer Symptome in Jugend- bzw. Erwachsenenalter sieht, auf eine Störung übertragen, die den Entwicklungsaspekt in den Vordergrund stellt?

Grundsätzlich stehen Untersuchungen zur Phänotypisierung von emotionalen Grundbedürfnissen, maladaptiven Schemata sowie Schemamodi bei Individuen mit HFA ganz am Anfang. Bislang gibt es keine klare Evidenz für die Entwicklung von Schemata und Bewältigungsmodi bei Menschen mit HFA. Deshalb bezieht sich der im Weiteren beschriebene Therapieansatz für Individuen mit HFA zwar auf das SchemaModus-Konzept, nutzt dieses jedoch auf eine neue Art und Weise als einen erweiterten Erklärungsansatz für zwischenmenschliche Kommunikation. Hierbei wird angenommen, dass Personen mit Autismus mentale Zustände anderer Personen und uneindeutiges Verhalten nur schwerlich erfassen können. Dies führt zu Problemen, wenn sie mit dem Bewältigungsverhalten nichtautistischer Personen konfrontiert werden, das gerade nicht direkt durch wahrnehmbare, situationale Reize verursacht wird. Demzufolge beabsichtigt der hier formulierte therapeutische Ansatz, über die Nutzung der Schema-Modus-Analy- 
se nichtautistischer Interaktionspartner ${ }^{1}$ eine Komplexitätsreduktion sozialer Situationen für Erwachsene mit HFA zu erreichen. Auf dieser Grundlage können Strategien entwickelt werden, wie Betroffene die wichtigsten Modi bei Interaktionspartnern erkennen und besser damit umgehen können.

\section{Prinzip der Intervention}

Grundlegend für das STISI bei Menschen mit HFA ist das oben eingeführte Schema-Modus-Modell, das Modi als aktivierte, komplexe Erlebenszustände und Handlungstendenzen beschreibt (Roediger 2016). Es werden Kindmodi, dysfunktionale internalisierte Elternmodi, maladaptive Bewältigungsmodi und derintegrierte Modus (gesunder Erwachsenenmodus) unterschieden. Ausdruck der Frustration emotionaler Grundbedürfnisse sind die Kindmodi, die als starke basale Emotionen auftreten. Daneben kommt es zu einer Aktivierung der sog. Innere-Eltern-Modi, die erlernte dysfunktionale Grundannahmen sowie verinnerlichte Bewertungsmuster umfassen und sich als „innere Kritiker" der Patienten zeigen. Auch wenn die Aktivierung der Kind- und InnereEltern-Modi intrasubjektiv stattfindet, haben beide Handlungsimpulse, die zu einem beobachtbaren Verhalten (Bewältigungsmodus) führen. Die Kind- und Elternmodi werden als primär angesehen, d.h. als noch zu explorierende innerliche Teilzustände auf der „hinteren Bühne“. Die Bewältigungsmodi werden als sekundäre Bewältigungsmuster des mit den Eltern- und Kindmodi verbundenen emotionalen Schmerzes auf der „Vorderen Bühne“ verstanden (Jacob und Arntz 2011). Genau diese sichtbaren Verhaltenstendenzen von Interaktionspartnern könnten für Menschen mit Autismus, die Schwierigkeiten haben, automatisch die Perspektive ihres Gegenübers $\mathrm{zu}$ übernehmen, wichtige Hinweise in Konfliktsituationen liefern.

1 Aus Gründen der besseren Lesbarkeit wird auf eine Nennung beider Geschlechter verzichtet. Sämtliche Personenbezeichnungen gelten sowohl für das weibliche als auch männliche Geschlecht.

Psychotherapeut 2018 63:235-242 https://doi.org/10.1007/s00278-018-0271-7

๑ Der/die Autor(en) 2018. Dieser Artikel ist eine Open-Access-Publikation.

H. Parpart · M. Krankenhagen · L. Albantakis · L. Henco · E. Friess · L. Schilbach

Schematherapie-informiertes, soziales Interaktionstraining. Interventionsansatz für Erwachsene mit hochfunktionalem Autismus

\section{Zusammenfassung}

Autismus-Spektrum-Störungen sind tiefgreifende Entwicklungsstörungen und durch Beeinträchtigungen der sozialen Interaktion und Kommunikation charakterisiert. Der hochfunktionale Autismus (HFA) ist ebenso durch soziale Schwierigkeiten gekennzeichnet, allerdings in Abwesenheit von intellektuellen Beeinträchtigungen. Personen mit HFA werden nicht immer im Kindes- und Jugendalter diagnostiziert, da sie aufgrund intakter intellektueller Fähigkeiten Kompensationsstrategien entwickeln. Im Gegensatz zu Betroffenen im Kindes- und Jugendalter weiß man wenig über die Effektivität der psychotherapeutischen Behandlung von Erwachsenen mit HFA. Verhaltenstherapeutische Konzepte richten sich v. a. nach Inhalten des sozialen Kompetenztrainings, jedoch ist unklar, ob dieser Ansatz tatsächlich sozial-interaktionelle Fertigkeiten verändert. In Anbetracht der vielversprechenden Erkenntnisse zur Effektivität der Schematherapie bei unterschiedlichen psychiatrischen Erkrankungen wird das
Schematherapie-informierte, soziale Interaktionstraining (STISI) für Erwachsene mit HFA vorgestellt. Eine zentrale Rolle spielt die Komplexitätsreduktion sozialer Situationen durch die Schema-Modus-Analyse und das Kennenlernen zugehöriger Indikatoren im Sozialverhalten nichtautistischer Personen bei den Betroffenen. Individuen mit HFA werden Verfahren zur Identifikation von Bewältigungsverhalten nichtautistischer Interaktionspartner vermittelt und interaktionell hilfreiche Handlungsmöglichkeiten aufgezeigt. Es wird angenommen und erste präliminäre Rückmeldungen von Patienten deuten darauf hin, dass Erwachsene mit HFA von dieser Strategie profitieren. Sie ermöglicht innen, schwierige soziale Situationen zu klären und damit negative Folgen zu verhindern.

\section{Schlüsselwörter}

Autismus-Spektrum-Störung · Coping-Verhalten · Soziale Kompetenz · Kommunikation . Gruppentherapie

\section{Schema therapy-informed social interaction training. Interventional approach for adults with high-functioning autism}

\section{Abstract}

Autism spectrum disorders are neurodevelopmental disorders, which are characterized by impairments of social interaction and communication. So-called high-functioning autism (HFA) is also characterized by these social difficulties, but in the absence of intellectual impairments. Individuals with HFA are not always diagnosed in childhood and adolescence, which might be due to compensatory strategies these individuals can develop, because of their intact intellectual capacities. In contrast to childhood and adolescence, very little is known about the effectiveness of psychotherapeutic treatment options for adults with HFA. Cognitive behavioral therapy suggests social competence training, but it is unclear whether this approach actually helps to change social interaction skills. In view of the promising findings on the effectiveness of schema therapy in various psychiatric disorders, we suggest that schema therapy-informed social interaction training (STISI) should be applied in patients with HFA. A fundamental idea is to reduce the complexity of social interactions by teaching patients with HFA to identify schema coping behavior in their non-autistic interaction partners and to learn ways of responding. It is hypothesized and first feedback from patients indicates that adults with HFA benefit from this strategy. It may help them to resolve interactional situations that could otherwise have negative consequences.

\section{Keywords}

Autism spectrum disorder - Coping behavior . Social competence - Communication - Group therapy 
Das Erkennen der Bewältigungsmodi so die hier vertretene Annahme - kann gelernt und darauf folgende Handlungsmöglichkeiten können erarbeitet und eingeübt werden.

Trotz Einschränkungen in der Kontaktfähigkeit und sozialen Anpassung wünschen sich die meisten Menschen mit ASS soziale Kontakte, Freundschaften oder auch eine Partnerschaft (Mazurek 2013). Ziel von STISI ist daher, soziale Kompetenzen zu stärken und über positive Erfahrungen mit sozialen Interaktionen für diese zu motivieren. Zunächst erfolgt vor sozialen Situationen eine Komplexitätsreduktion „nach innen“, indem die eigenen Ziele und Erwartungen an die andere Person überprüft werden. Darauf folgt eine Komplexitätsreduktion "nach außen": Im Vordergrund steht hierbei nicht, spezifische Verhaltensweisen für bestimmte soziale Situationen (wie z. B. Telefonieren oder Small Talk) einzuüben, sondern vielmehr anhand von sozialen Analysefaktoren zu lernen, das Verhalten der Interaktionspartner schneller einzuschätzen und damit eine größere Flexibilität gerade in schwierigen sozialen Situationen zu erleben. So wird ein Verständnis für interaktionelle Schwierigkeiten erreicht, der Handlungsspielraum erweitert und das Selbstwertgefühl gestärkt. Wird in sozialen Situationen weniger Frustration und Hilflosigkeit erlebt, kommt es seltener zu sozialem Rückzug. Neben der Behandlung der Kernsymptomatik wird damit auch eine Linderung der komorbiden Symptomatik (z. B. soziale Ängste oder Depression) angestrebt. So können die Lebensqualität erhöht und berufliche Chancen verbessert werden.

\section{Überprüfen eigener Ziele und Erwartungen}

Menschen mit HFA berichten häufig von einer Überforderung in sozialen Interaktionen, die Handlungsunfähigkeit oder z. T. impulsive Verhaltensweisen zur Folge hat. Ein wichtiger Schritt hin zu einer Vereinfachung der sozialen Konfliktsituation ist die Überprüfung der eigenen Ziele in Bezug auf die Interaktion und die eigenen Erwartungen an den Interaktionspartner. So gilt es beispielsweise vor einer Small-Talk-Situation zu überprüfen, ob Interesse an einer Fortsetzung der Kommunikation mit der anderen Person besteht und somit eine reibungslose Interaktion angestrebt wird, oder ob aufgrund von zu hohem Ressourcenverbrauch und Mangel an Interesse an der Interaktion ein Verlassen der Situation zielführender ist. Dieser erste Schritt entspricht einer Komplexitätsreduktion nach innen. Ein größeres Verständnis für interaktionelle Schwierigkeiten wird im zweiten Schritt über die Analyse der Bewältigungsmodi des Interaktionspartners angestrebt (Komplexitätsreduktion nach außen).

\section{Bewältigungsmodi}

\section{Konzept}

Zentral bei der Schema-Modus-Analyse sind die dysfunktionalen Bewältigungsmodi, die auf Spannungsreduktion und Konfliktbewältigung abzielen. Mit der Annahme, dass Menschen versuchen, schwierige Situationen nach ähnlichen Prinzipien zu bewältigen, die auch bei Tieren zu finden sind, macht sich die ST das biologische Modell der Verhaltensprägung zunutze. In Anlehnung an das Tiermodell beobachtbarer Reaktionsmuster in Konflikten werden folglich 3 Bewältigungsstile formuliert: Unterordnung, Vermeidung und Überkompensation. Im Bewältigungsstil Unterordnung verhält sich der Betroffene so, als sei das Schema wahr. Er geht einher mit einem starken Spüren schemaassoziierter Affekte, Angepasstsein, unterwürfigem Auftreten sowie einem fehlenden Fokus auf eigene Bedürfnisse. Personen unterwerfen sich, um Konflikte zu vermeiden („surrender" oder "follow"). Vermeidung zeigt sich im Umgehen sozialer Situationen und/oder Emotionen entweder durch Rückzug und passives Erstarren, um nicht gesehen zu werden („Flucht nach innen“ bzw. „freeze“) oder durch Aktionismus als Flucht nach außen (,flight“), um einer belastenden Situation zu entfliehen. Der vermeidende Bewältigungsstil ist meist im Kontakt nicht spürbar, da schemarelevante Affekte und Kontakte gescheut werden. Im Zuge des Bewältigungsstils Überkompensation verhält sich der Betroffene so, als sei das Gegenteil des Schemas wahr. Ist eine Person in der aktiv-kämpfenden Überkompensation („fight“), fühlt sich das Gegenüber meist dominiert oder bedroht. $\mathrm{Zu}$ beachten ist, dass Personen meist mehrere Bewältigungsmodi im Wechsel verwenden.

Bewältigungsmodi veranschaulichen die zwischenmenschlichen Beziehungen: Personen unterwerfen sich, um ihr Bindungsbedürfnis zu befriedigen, gehen aus dem Kontakt, um soziale Konflikte zu vermeiden, oder treten dominant auf, um zu kontrollieren. Über die 3 Grundkategorien liegt den Bewältigungsmodi folglich eine interpersonale Definition von Beziehung zugrunde (Roediger 2016).

\section{Identifikation}

Damit Personen mit HFA die Bewältigungsstile und Moduswechsel ihrer nichtautistischen Interaktionspartner erkennen lernen, werden im nächsten Schritt von STISI beobachtbare Indikatoren der 3 Coping-Stile vermittelt. Relevant sind nonverbale Kommunikationssignale wie Blickkontakt und -richtung, Gesichtsausdruck, körperliche Nähe und Abstand zum Gegenüber, Körperhaltung und spezifische Gesten sowie explizite, sprachlich vermittelte Signale wie Sprechgeschwindigkeit, Intonation, Lautstärke, Betonung, Tonfall, Aussprache, Sprechpausen und Stimmlage. Im Gruppensetting wird die Erkennung dieser Indikatoren über Videoanalysen nichtautistischer Personen, die sich in Interaktion mit einer anderen nichtautistischen Person befinden, trainiert. Nachdem die Coping-Stile identifiziert und unterschieden werden können, sollten im zweiten Schritt mögliche Gründe für das Bewältigungsverhalten des Interaktionspartners gemeinsam mit den Teilnehmern erarbeitet werden. Dabei steht nicht im Fokus, die dahinterliegenden Schemata der Interaktionspartner bzw. deren motivationale Anteile zu bestimmen, sondern vielmehr über die Kategorisierung des Verhaltens anhand einer Modusanalyse ein Verständnis gerade für problematische emotionale Zustände des Gegenübers zu entwickeln. Dies ist insbesondere für die Klärung der eigenen Bedürfnisse in Bezug auf die Gestaltung der Kommunikation und damit 
Tab. 1 Sitzungsübersicht der Münchner Autismus-Therapiegruppe für Erwachsene

\begin{tabular}{l|l}
\hline $\begin{array}{l}\text { Sitzungs- } \\
\text { nummer }\end{array}$ & Sitzungsinhalt \\
\hline 1 & Einführung und Kennenlernen \\
\hline 2 & Autismus-Spektrum-Störungen (ASS) und Begleiterscheinungen/-erkrankungen \\
\hline 3 & Emotionen, Selbstwahrnehmung und Entspannung \\
\hline 4 & Stress: Modell und Ursachen \\
\hline 5 & Stressbewältigung über Situationsanalysen \\
\hline 6 & STISI I: Einführung in das Konzept der Schematherapie \\
\hline 7 & STISI II: Eigene Ziele und Erwartungen an den Interaktionspartner \\
\hline 8 & STISI III: Identifikation von Bewältigungsmodi 1 \\
\hline 9 & STISI IV: Identifikation von Bewältigungsmodi 2 \\
\hline 10 & STISI V: Erarbeiten von Handlungsmöglichkeiten 1 \\
\hline 11 & STISI VI: Erarbeiten von Handlungsmöglichkeiten 2 \\
\hline 12 & Stärken eigener Ressourcen \\
\hline STISI Schematherapie-informiertes, soziales Interaktionstraining \\
\hline
\end{tabular}

für die Motivation der darauffolgenden eigenen Handlung relevant.

\section{Erarbeiten von \\ Handlungsmöglichkeiten}

Individuen mit HFA sind in komplexen sozialen Situationen, gerade in der direkten Interaktion häufig überfordert und verhalten sich daher passiv und vermeidend. Wird im Zuge des Interaktionstrainings das Bewältigungsverhalten eines Interaktionspartners von einer Person mit HFA durch unterschiedliche Analysefaktoren erkannt, können anschließend an die eigene Motivation angepasste Handlungsmöglichkeiten erarbeitet werden, um die Entstehung eines Beziehungskonflikts zu verhindern. $\mathrm{Zu}$ betonen ist, dass nicht eine soziale Anpassung unter Vernachlässigung eigener Interessen angestrebt wird. Vielmehr werden flexible, situationsangemessene und für die Teilnehmer nachvollziehbare Verhaltensweisen erarbeitet und trainiert. Hierzu bieten sich unterschiedliche Methoden an - wie beispielsweise Videoanalysen der Teilnehmer in Small-TalkSituationen und anschließende Rollenspiele -, die auf den individuellen Bedarf der Betroffenen abgestimmt werden können. Im Vordergrund stehen die aktive Rolle jedes Teilnehmers, das Erleben direkter, sozialer Interaktion sowie die Aneignung verbaler und nonverbaler Kommunikationssignale.

\section{Gruppentherapeutisches Setting}

Das STISI ist im Gruppensetting anzuleiten, da soziale Fertigkeiten direkt in der Interaktion mit den anderen Gruppenteilnehmern mit Autismus in einem geschützten Rahmen erlernt werden können. Gruppenspezifische Wirkfaktoren wie beispielsweise die Erfahrung von Zugehörigkeit (Yalom und Leszcz 2005) können außerdem einen positiven Einfluss haben. So entstehen positiv bewertete soziale Kontakte, die auch häufig nach Beenden der Gruppe weitergeführt werden. Zu schematherapeutischer Gruppentherapie existieren bereits einige Ansätze (z.B. Farrell und Shaw 2013; Simpson et al. 2010; van Vreeswijk et al. 2014), jedoch findet sich bislang keine therapeutische Verwendung des Konzepts für Erwachsene mit HFA.

\section{Evaluation}

In 2 Pilotgruppen der Münchner Autismus-Therapiegruppe für Erwachsene (MATE, Sitzungsübersicht: • Tab. 1) am Max-Planck-Institut für Psychiatrie München wurden 2016 erste Erfahrungen mit STISI gesammelt. Die Gruppen wurden von 2 Diplom-Psychologinnen in psychotherapeutischer Weiterbildung mit Schwerpunkt Verhaltenstherapie geleitet und pilotevaluiert. Alle Teilnehmer wurden zuvor in der Ambulanz für Störungen der sozialen Interaktion des MaxPlanck-Instituts für Psychiatrie München
Tab. 2 Demografische Daten der Stich-

probe

Merkmal

Ausprägung

Geschlecht (Anzahl, $n$ )

- Männlich 5

- Weiblich 4

Alter (Jahre; $M \pm S D$ ) $\quad 31,1 \pm 13,8$

Verbaler Intelligenzquotient $\quad 109,4 \pm 13,3$

$(\mathrm{M} \pm \mathrm{SD})$

Autismus-Quotient (M \pm SD) $\quad 35,3 \pm 7,1$

$M$ Mittelwert, SD Standardabweichung

diagnostiziert und hatten entweder die Diagnose „Asperger-Syndrom“ (F84.5) oder „HFA“ (F84.0, IQ > 70) vorliegen. In die Auswertung wurden nur Patienten eingeschlossen, die nicht mehr als 2-mal fehlten. In - Tab. 2 sind die demografischen Daten der Stichprobe dargestellt. Zur Evaluation wurden der Stundenbogen für die allgemeine und differenzielle Einzelpsychotherapie (STEP, Krampen 2002) sowie ein kurzer MATE-Feedbackbogen verwendet. Der STEP erfasst das Erleben allgemeiner Wirkfaktoren einer Psychotherapie und beinhaltet folgende 3 inhaltlich komplementäre Subskalen zu der in einer Sitzung erlebten

1. motivationalen Klärung,

2. aktiven Hilfe zur Problembewältigung,

3. therapeutischen Beziehung.

$\mathrm{Zu}$ diesen 3 Subskalen wurden T-Werte ermittelt, die im Vergleich zu einzelpsychotherapeutischen Langzeitbehandlungen im Durchschnittsbereich liegen (- Abb. 1). Im Vergleich zu psychoedukativen Elementen bei ASS und Depression sowie Inhalten und Übungen zur Stressbewältigung wurden die STISI-Sitzungen sowie die letzte Sitzung zu eigenen Ressourcen als am hilfreichsten bewertet. Die T-Werteliegen im Vergleich zu einzelpsychotherapeutischen Langzeitbehandlungen im Durchschnittsbereich (• Abb. 2).

In Anlehnung an den GATE(Gruppentraining für Autismus im Erwachsenenalter)-Evaluationsbogen (Gawronski et al. 2012) wurde für eine deskriptive Prozessdiagnostik ein MATE-Feedbackbogen an die Teilnehmer ausgeteilt. Die 5 Items sollten auf einer 5-stufigen LikertSkala eingeschätzt werden. Die Ergeb- 

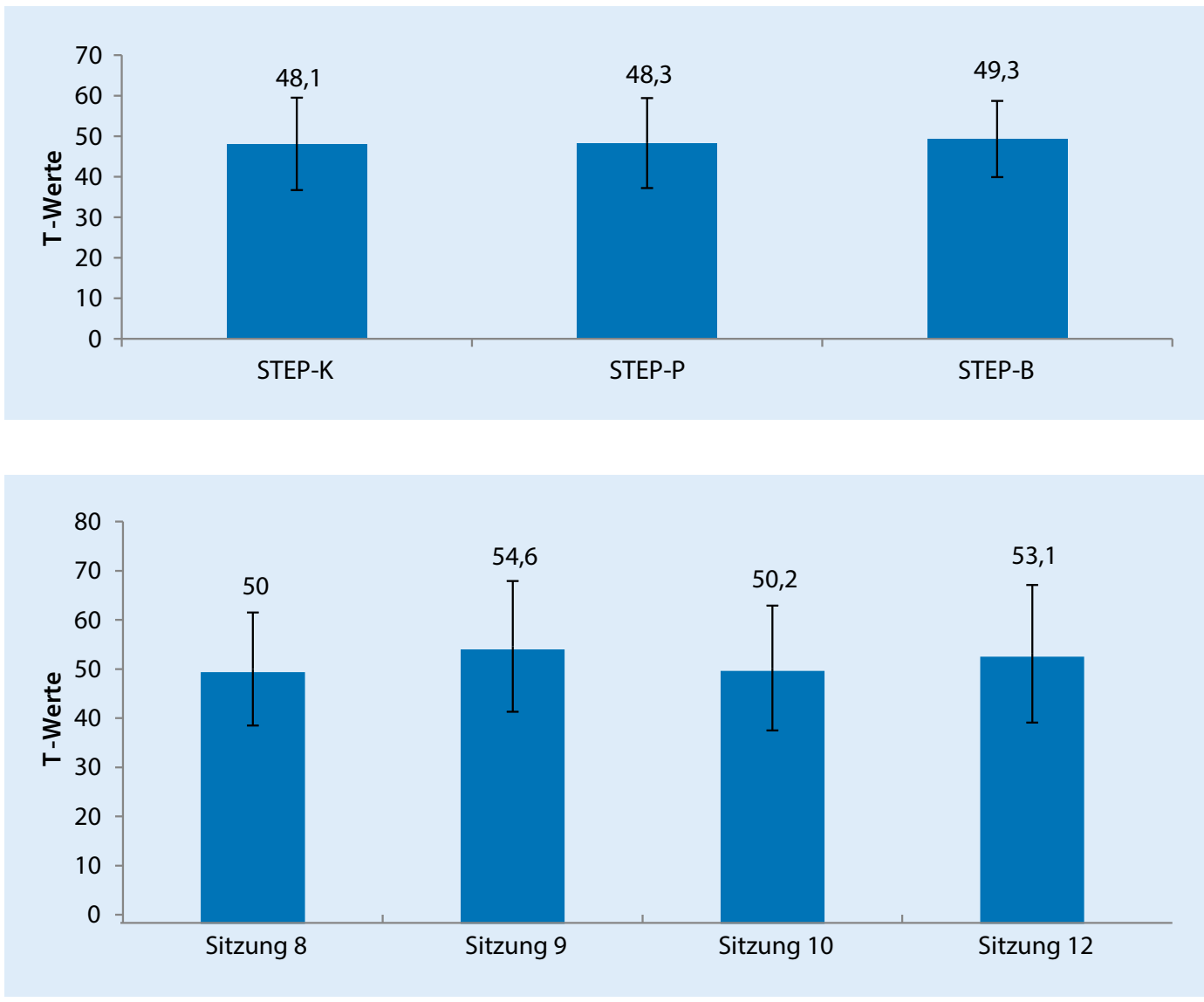

Abb. $1<$ Bewertung der Wirkfaktoren der psychotherapeutischen Behandlung. Gemittelte T-Werte aller Teilnehmer hinsichtlich der 3 Subskalen des STEP. STEP-K motivationale Klärung, STEP-P aktive Hilfe zur Problembewältigung, $S T E P-B$ therapeutische Beziehung

Abb. $\mathbf{2} \triangleleft$ Bewertung der hilfreichsten Sitzungen des Therapieprogramms. Gemittelte T-Werte aller Teilnehmer bezüglich der am positivsten bewerteten Sitzungen. Sitzung 8 „STISI III: Identifikation von Bewältigungsmodi 1", Sitzung 9 "STISI IV: Identifikation von Bewältigungsmodi 2", Sitzung 10 "STISI V: Erarbeiten von Handlungsmöglichkeiten 1 ", Sitzung 12 "Stärken eigener Ressourcen"

Abb. $3<$ Sitzungsevaluation. Mittlere Rohwerte aller Teilnehmer $(n=9)$ bezüglich der 4 Items des Fragebogens. Item 1 "Ich habe mich in der Gruppe heute wohl gefühlt." Item 2 "Die Inhalte waren hilfreich für mich." Item 3 „Ich bin gespannt auf die neue Sitzung." Item 4 „Die Therapeuten sind angenehm und kompetent." (Skala: 1: stimmt überhaupt nicht, 5: stimmt vollkommen) nisse der mittleren Bewertungen für die ersten 4 Items sind in $\mathbf{A b b} \mathbf{~} \mathbf{3}$ abgebildet.

Weiterhin wurde mit Item 5 erhoben, ob sich das Interesse, sich mit anderen Menschen zu treffen, im Zuge des Gruppentrainings verändert. Es zeigte sich ein signifikanter Unterschied von Sitzung 2 zu Sitzung 12 (• Abb. 4).

Die Ergebnisse der Pilotevaluation deuten auf eine hohe Akzeptanz des Interaktionstrainings bei den Teilnehmern hin. Eine genauere Evaluation steht aufgrund der bisher begrenzten Teilnehmerzahl jedoch noch aus.

\section{Ausblick}

Um die klinische Effektivität des Trainings bewerten zu können, sind randomisierte kontrollierte Evaluationsstudien geplant. Im Vergleich zur STISI-Studiengruppe soll eine Kontrollgruppe, die als Intervention das soziale Kompetenztraining erhält, etabliert werden. Neben qualitativen Fragebogenevaluationen sollen auch objektive und belastbare Outcome-Variablen identifiziert und gemessen werden. Durch unabhängiges klinisches Fachpersonal soll die Erfassung des allgemeinen Funktionsniveaus anhand der Skala Global Assessment of Functioning (GAF), des sozialen und beruflichen Funktionsniveaus anhand der Social and Occupational Functioning Assessment Scale (SOFAS) sowie der subjektiven Lebensqualität anhand der 


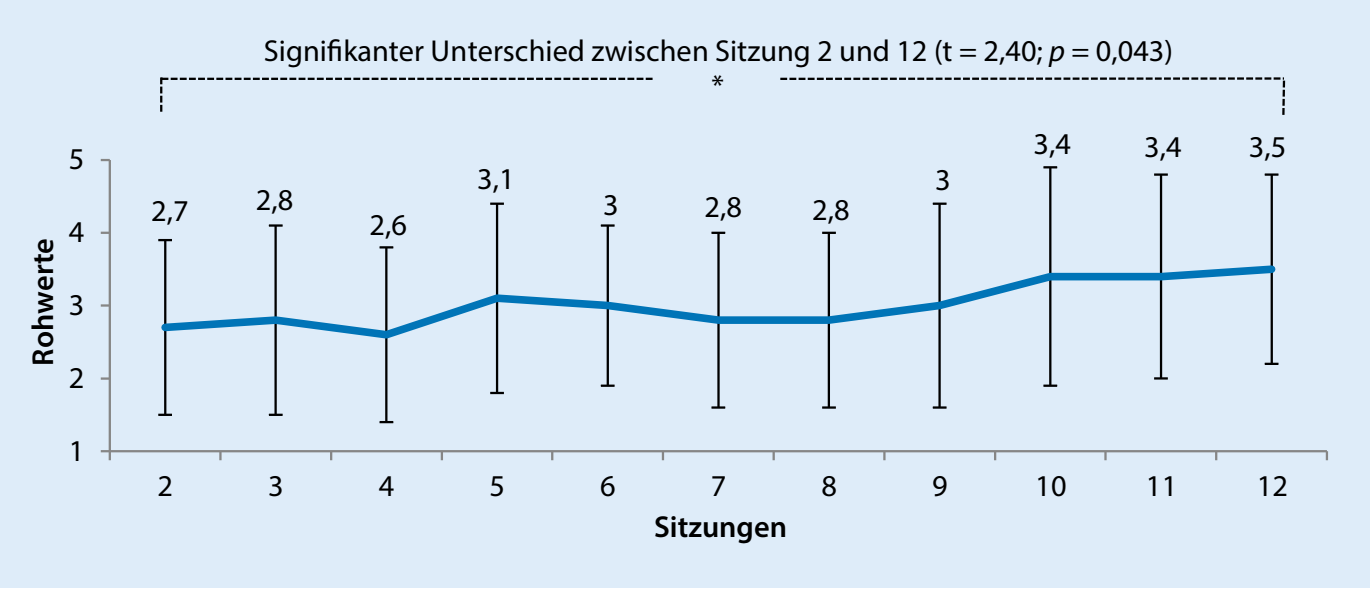

Abb. $4<$ Effekte der Therapie auf die Bereitschaft zu sozialen Interaktionen. Gemittelte Rohwerte aller Teilnehmer für Item 5 (,Ilch habe Interesse, mich mit Freunden, Bekannten oder Kollegen zu treffen."), $p \mathrm{Si}$ gnifikanzniveau, $t$ Prüfgröße (Skala: 1: stimmt überhaupt nicht, 5: stimmt vollkommen)

Skala The World Health Organization Quality of Life (WHOQOL) gewährleistet werden. Zukünftige Untersuchungen sehen außerdem eine Validierung der empirischen Indikatoren der 3 CopingStile vor. Dafür könnten Sprachanalysen, „Eye-tracking“-Studien zu sozialem Blick oder Bewegungsanalysen bei nichtautistischen Personen hilfreich sein, um Parameter auszumachen, die einen Moduswechsel anzeigen. Weiterhin sind Studien mit interaktionsbasierten hirnbildgebenden Verfahren geplant, um diejenigen Personen mit Autismus $\mathrm{zu}$ identifizieren, die besonders von STISI profitieren. Auf diese Weise werden die Effekte von Psychotherapie neurowissenschaftlich validiert, und es kann über die Ermittlung von Biomarkern eine objektive Grundlage geschaffen werden. Auch eine Kombination von STISI mit psychopharmakologischer Intervention wird angestrebt und könnte auf diesem Weg entsprechend untersucht werden. So fördert Oxytozin in seiner Doppelrolle als Hormon und als Neurotransmitter nicht nur auf peripherem Weg soziale Bindung, sondern beeinflusst auch zentralnervöse sozial-kognitive Informationsverarbeitung und Verhaltensweisen (Groppe et al. 2013; Guastella et al. 2008; Scheele et al. 2013). Ferner könnten diese Ergebnisse durch eine Fokussierung derjenigen interaktionsbasierten Aspekte, die funktionell hirnbildgebend als therapeutische Biomarker ausgemacht werden, zu einer weiteren Verbesserung von STISI führen. Insgesamt könnte dieser Ansatz helfen, die Wahl der Behandlungsmöglichkeiten zur Verbesserung sozial-interaktioneller Fähigkeiten bei Personen mit Autismus differenziert auf das Individuum abzustimmen.

\section{Fazit für die Praxis}

- Zu verhaltenstherapeutischen Programmen im Gruppensetting stellt das Schema-Modus-Konzept möglicherweise eine wertvolle Erweiterung dar, um soziale Interaktion und soziale Konflikte für Erwachsene mit HFA zu erklären und soziale Kompetenzen zu verbessern. Eine zentrale Rolle spielt die Komplexitätsreduktion sozialer Situationen durch die Analyse von Bewältigungsmodi bei nichtautistischen Interaktionspartnern.

- Die bisherigen Erfahrungen mit dem STISI als Psychoedukations- und Trainingsansatz sind vielversprechend.

- Für die empirische Validierung des vorgelegten Konzepts sind noch Wirksamkeitsnachweise über randomisierte kontrollierte Evaluationsstudien, die Überprüfung der Wirkung auf Alltagsfunktionen sowie Grundlagenstudien zur Bedeutung der Bewältigungsmodi geplant.

\section{Korrespondenzadresse}

\section{H. Parpart}

Unabhängige Max-Planck-Forschungsgruppe für Soziale Neurowissenschaft, Max-PlanckInstitut für Psychiatrie

Kraepelinstr. 2-10, 80804 München,

Deutschland

hella_parpart@psych.mpg.de
Danksagung. Hella Parpart dankt der BarbaraWengeler-Stiftung für ihre Unterstützung.

Funding. Open access funding provided by Max Planck Society.

\section{Einhaltung ethischer Richtlinien}

Interessenkonflikt. H. Parpart, M. Krankenhagen, L. Albantakis, L. Henco, E. Friess und L. Schilbach geben an, dass kein Interessenkonflikt besteht.

Dieser Beitrag beinhaltet keine von den Autoren durchgeführten Studien an Menschen oder Tieren.

Open Access. Dieser Artikel wird unter der Creative Commons Namensnennung 4.0 International Lizenz (http://creativecommons.org/licenses/by/4.0/deed. de) veröffentlicht, welche die Nutzung, Vervielfältigung, Bearbeitung, Verbreitung und Wiedergabe in jeglichem Medium und Format erlaubt, sofern Sie den/die ursprünglichen Autor(en) und die Quelle ordnungsgemäßnennen, einen Linkzur Creative Commons Lizenz beifügen und angeben, ob Änderungen vorgenommen wurden.

\section{Literatur}

American Psychiatric Association (2013) Diagnostic and statistical manual of mental disorders, 5. Aufl. American Psychiatric Association, Washington, DC

Bamelis L, Bloo J, Bernstein D, Arntz A (2015) Effectiveness studies. In: Van Vreeswijk M, Broersen J, Nadort M (Hrsg) The Wiley-Blackwell handbook of schema therapy: theory, research, and practice. John Wiley \& Sons, New York, S 493-510

Barazandeh H, Kissane DW, Saeedi N, Gordon M (2016) A systematic review of the relationship between early maladaptive schemas and borderline personality disorder/traits. Pers Individ Dif 94:130-139

Beck AT, Freeman A, Davis DD (2004) Cognitive therapy of personality disorders. Guilford, New York

Bortolon C, Capdevielle D, Boulenger JP, GelyNargeot MC, Raffard S (2013) Early maladaptive schemas predict positive symptomatology 
in schizophrenia: a cross-sectional study. Psychiatry Res 209:361-366

Developmental DMNSY, 2010 Principal Investigators (2014) Prevalence of autism spectrum disorder among children aged 8 years-autism and developmental disabilities monitoring network 11 sites, United States, 2010. MMWR Surveill Summ 63:1-21

Ebert D, Fangmeier T, Lichtblau A, Peters J, BiscaldiSchäfer M, van Elst LT (2013) AspergerAutismus und hochfunktionaler Autismus bei Erwachsenen: Ein Therapiemanual der Freiburger Autismus-Studiengruppe. Hogrefe, Göttingen

Farrell J, Shaw I (2013) Schematherapie in Gruppen. Therapiemanual für die Borderline-Persönlichkeitsstörung. Beltz, Weinheim

Frazier TW, Youngstrom EA, Speer L, Embacher R, Law P, Constantino J, Eng C (2012) Validation of proposed DSM-5 criteria for autism spectrum disorder. J Am Acad Child Adolesc Psychiatry 51:28-40

Gaus VL (2007) Cognitive-behavioral therapy for adult Asperger syndrome. Guilford, New York

Gawronski A, Pfeiffer K, Vogeley K (2012) Hochfunktionaler Autismus im Erwachsenenalter. Verhaltenstherapeutisches Gruppenmanual. Beltz, Weinheim

Groppe SE, Gossen A, Rademacher L, Hahn A, Westphal L et al (2013) Oxytocin influences processing of socially relevant cues in the ventral tegmental area of the human brain. Biol Psychiatry 74:172-179

Guastella AJ, Mitchell PB, Dadds MR (2008) Oxytocin increases gaze to the eye region of human faces. Biol Psychiatry 63:3-5

Hayes SC (2004) Acceptance and commitment therapy, relational frame theory, and the third wave of behavioral and cognitive therapies. Behavior Therapy 35(4):639-665

Jacob G, Arntz A (2011) Schematherapie in der Praxis. Beltz, Weinheim

Kamp-Becker I, Smidt J, Ghahreman M, HeinzelGutenbrunner M, Becker K, Remschmidt H (2010) Categorical and dimensional structure of autism spectrum disorders: the nosologic validity of Asperger syndrome. J Autism Dev Disord 40:921-929

Kim JE, Lee SW, Lee SJ (2014) Relationship between early maladaptive schemas and symptom dimensions in patients with obsessive-compulsive disorder. Psychiatry Res 215:134-140

Kim YS, Leventhal BL, Koh YJ, Fombonne E, Laska E, Lim EC, Grinker RR (2011) Prevalence of autism spectrum disorders in a total population sample. Am J Psychiatry 168:904-912

Krämer K, Gawronski A, Vogeley K (2016) Zur Diagnostik und Behandlung von AutismusSpektrum-Störungen im Erwachsenenalter. Fortschr Neurol Psychiatr 84:578-588

Krampen G (2002) STEP. Stundenbogen für die Allgemeine und Differenzielle Einzelpsychotherapie. Hogrefe, Göttingen

Lehnhardt FG, Gawronski A, Volpert K, Schilbach L, Tepest R, Huff W, Vogeley K (2011) AutismusSpektrum-Störungen im Erwachsenenalter: klinische und neuropsychologische Befunde spätdiagnostizierter Asperger-Syndrome. Fortschr Neurol Psychiatr 79:290-297

Lehnhardt FG, Gawronski A, Pfeiffer K, Kockler H, Schilbach L, Vogeley K (2013) The investigation and differential diagnosis of Asperger syndrome in adults. Dtsch Arztebl Int 110:755-763
Lugnegård T, Hallerbäck MU, Gillberg C (2011) Psychiatric comorbidity in young adults with a clinical diagnosis of Asperger syndrome. Res Dev Disabil 32:1910-1917

Lyall K, Croen LA, Daniels J, Fallin MD, Ladd-Acosta C, Lee BK, Newschaffer CJ (2017) The changing epidemiology of autism spectrum disorders. Annu Rev Public Health 38:81-102

Mazurek MO (2013) Loneliness, friendship, and wellbeing in adults with autism spectrum disorders. Autism 18:223-232

Nilsson KK, Nielsen StraarupK, Halvorsen M(2015) Early maladaptive schemas: a comparison between bipolar disorder and major depressive disorder. Clin Psychol Psychother 22:387-391

Remschmidt H, Kamp-Becker I (2006) AspergerSyndrom. Springer, Heidelberg

Roediger E (2016) Schematherapie: Grundlagen, Modell und Praxis. Schattauer, Stuttgart

Scheele D, Wille A, Kendrick KM, Stoffel-Wagner B, Becker B et al (2013) Oxytocin enhances brain reward system responses in men viewing the face of their female partner. Proc Natl Acad Sci U SA 110:20308-20313

Schilbach L (2016) Toward a second-person neuropsychiatry. Philos Trans RSoc B 371:20150081

Simpson SG, Morrow E, van Vreeswijk M, Reid C (2010) Group schema therapy for eating disorders: a pilot study. Front Psychol 16:182

van Vreeswijk MF, Spinhoven P, Eurelings-Bontekoe $\mathrm{EH}$, Broersen J (2014) Changes in symptom severity, schemas and modes in heterogeneous psychiatric patient groups following short-term schema cognitive-behavioural group therapy: a naturalistic pre-treatment and post-treatment design in an outpatient clinic. Clin Psychol Psychother 21:29-38

Weltgesundheitsorganisation (2013) Internationale Klassifikation psychischer Störungen: ICD-10 Kapitel V (F) Klinisch-diagnostische Leitlinien, 4. Aufl. Huber, Bern

Yalom ID, Leszcz M (2005) Theory and practice of group psychotherapy. Basic Books, New York

Young JE, Klosko JS, Weishaar ME (2008) Schematherapie. Ein praxisorientiertes Handbuch. Junfermann, Paderborn

\section{Psychotherapie - Was für wen?}

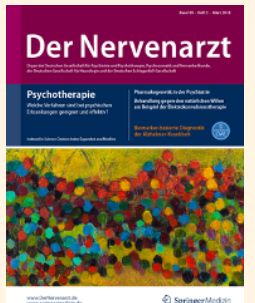

Die Anwendung psychotherapeutischer Verfahren ist bei der Behandlung psychischer Erkrankungen nicht nur empfehlenswert, sondern in vielen

Fällen unverzichtbar. Eine wissenschaftlich fundierte Antwort auf die Frage der individuellen Passung bleibt aber - sowohl hinsichtlich Datenlage als auch Komplexität der Thematik - derzeit noch eine Herausforderung. Umso mehr dienen die in Der Nervenarzt 03/2018 umfassend und damit auch wegweisend bearbeiteten Leitthemenbeiträge Ihrer Orientierung für den Klinik- und Praxisalltag, insbesondere unter den Aspekten Wirksamkeit und differentielle Indikationsstellung.

- Depressive Störungen - Verfahren, Evidenz und Perspektiven

- Psychotherapie bei bipolaren Störungen und Angsterkrankungen

- Differentialindikation für die Psychotherapie bei Psychosen

- Evidenzbasierte Psychotherapie bei Abhängigkeitserkrankungen

Suchen Sie noch mehr zum Thema? Mit e.Med - den maßgeschneiderten Fortbildungsabos von Springer Medizin - haben Sie Zugriff auf alle Inhalte von SpringerMedizin.de. Sie können schnell und komfortabel in den für Sie relevanten Zeitschriften recherchieren und auf alle Inhalte im Volltext zugreifen.

Weitere Infos zu e.Med finden Sie auf springermedizin.de unter "Abos" 